\title{
The Basic Reproduction Ratio for Sexually Transmitted Diseases \\ Part 2. Effects of Variable HIV Infectivity
}

\author{
K. DIETZ
}

Institut für Medizinische Biometrie, Universität Tübingen, Germany

J. A. P. HEESTERBEEK

CWI, Amsterdam, The Netherlands

AND

D. W. TUDOR*

Institut für Medizinische Biometrie, Universität Tübingen, Germany

Received 8 November 1992; revised 12 December 1992

\begin{abstract}
In this paper we investigate the effects of variable infectivity on the spread of HIV in a heterosexual population where pair formation and separation are taken into account. We calculate the basic reproduction ratio as a function of the number of new partners during the infectious period, keeping the total number of contacts fixed. Numerical evidence suggests that the basic reproduction ratio decreases for variable infectivity if the average infectivity is kept constant.
\end{abstract}

\section{INTRODUCTION}

In a previous paper [3] we described how to calculate the basic reproduction ratio $R_{0}$ for infectious disease models where an arbitrary but finite number of infection states are recognized and the processes of pair formation and separation are taken into account. The model in [3] is a pair formation analog of a multistage variable infectiousness model first developed by Blythe and Anderson [1] and Jacquez et al. [6], and a generalisation of [2]. In the present paper we apply that method of calculation to study the effects on the spread of HIV of variable infectivity. Remember that, because we want to calculate $R_{0}$, we only

* Present address: Synthélabo Recherche (L.E.R.S.), 31 ave Paul VaillantCouturier, 92225 Bagneux, France. 
keep track of the partnerships of infected individuals formed during their remaining lifetime after they become infected, and we may assume that all new partners are susceptible [2].

\section{DESCRIPTION OF MODEL AND CALCULATION OF $R_{0}$}

We start this section by giving a concise description of the model assumptions in [3] and indicating which modifications and concretizations were carried out for the present paper.

In [3] every pair initially passed through a courtship period characterized by the absence of sexual contacts. Given that the pair had not yet separated, the first sexual contact marked the end of the courtship period and the beginning of the sexually active phase. For the sake of simplicity, we neglect the courtship period in the present paper, that is, we take it to be infinitely short. This implies that a pair is initiated by a sexual contact.

We recognize the following characteristics of an infected individual:

$$
\begin{aligned}
& \text { Infection state: } \quad i \in\{1, \ldots, n\}, \\
& \text { Partnership state: } \quad j \in\{-1,0,1, \ldots, n\} .
\end{aligned}
$$

Here -1 means that the individual is single (no partner at the moment of observation); 0 means that the individual is paired with a susceptible; partnership state $j \in\{1, \ldots, n\}$ means that the individual is paired with an infected individual who has infection state $j$. Together the two characteristics determine the type $(i, j)$ of an infected individual. For subsequent numerical calculations we choose $n=4$. The infection states 1-4 are assumed to correspond to the possible phases in the development of HIV infection: an initial burst of infectiousness, followed by a (possibly long) period of virtual noninfectiousness, then a new infectious period in a pre-AIDS phase, and finally the disease phase of full-blown AIDS.

Now we extend our notation by taking the male/female dichotomy into account. We indicate the sex of an individual by $k \in\{1,2\}$, where 1 indicates male and 2 indicates female. We restrict our attention to heterosexual contacts. The type of an infected individual is then written as

$$
(i, j ; k), \quad i \in\{1, \ldots, 4\} ; j \in\{-1,0, \ldots, 4\} ; k \in\{1,2\}
$$


where $k$ is the sex of the individual, who has infection state $i$ and partnership state $j$. Singles are of type $(i,-1 ; k)$.

We make the following assumptions:

(1) The infection states are passed through in natural order, always beginning with state 1 . Thus, a newly infected individual of sex $k$ whose partner is in infection state $j$ has the type $(1, j ; k)$.

(2) $\mu_{0}$ is the death rate of susceptible males and females; $\mu_{i}$ is the death rate of infected male or female individuals with infection state $i$.

(3) The transition rates from state $i$ to state $i+1, i=1,2,3$, are denoted by $\theta_{i}$. Then the time spent in infection state $i$ is exponentially distributed with parameter $\theta_{i}+\mu_{i}$.

(4) The infectivity of an individual of sex $k$ in infection state $i$ is described by the probability $p_{i}(k)$ that an unprotected sexual contact with a susceptible of the opposite sex leads to transmission.

(5) Every single individual of sex $k$ with infection state not equal to 4 has a constant rate $\rho(k)$ of acquiring new partners. We assume that individuals in infection state 4 do not establish new sexual relationships. These rates must satisfy the consistency condition $\rho(1) x(1)=\rho(2) x(2)$, where $x(k)$ denotes the number of susceptibles of sex $k$ in the population. In other words, the ratio of the partner acquisition rates equals the reciprocal of the sex ratio in the susceptible population. The divorce rate is $\sigma$. We assume that each time an infected individual in infection state $i, i \in\{1,2,3,4\}$, with a partner in state $j, j \in\{0, \ldots, 4\}$, becomes single, either by divorce or by the partner's death, there is a probability $s_{i j}$ that this individual stays sexually active (i.e., a probability $1-s_{i j}$ of becoming permanently sexually inactive).

(6) By definition a partnership starts with one sexual contact.

(7) Following the initiating contact, there are $\beta$ sexual contacts per unit of time during the partnerships if none of the partners has infection state 4 . If one of the partners has infection state 4 , we assume that there are no sexual contacts.

Let $\Lambda:=\{(i, j ; k): 1 \leqslant i \leqslant 4,-1 \leqslant j \leqslant 4, k \in\{1,2\}\}$ be the set of all possible types. Then $|\Lambda|=48$ and consequently our type space is $\mathbb{R}^{48}$. Let $\Sigma:=\{1,2, \ldots, 48\}$. We will call $\Sigma$ the state space of infectives, and the elements of $\Sigma$ are called states. Let $L: \Lambda \rightarrow \Sigma$ describe the ordering on $\Lambda$ where we first take the lexicographic order of the types that an infected male can have and subsequently the lexicographic order of the female types.

We describe the changes in infection state and partnership state of male and female individuals as a Markov process on $\Sigma$. Let the matrix $G: \mathbb{R}^{48} \rightarrow \mathbb{R}^{48}$ describe the transition rates between the states; that is, 
$g_{r s}$ gives the rate of leaving state $s \in \Sigma$ to go to state $r \in \Sigma$. On the basis of the assumptions stated above, $G$ is given by

$$
G=\left(\begin{array}{cc}
G(1) & 0 \\
0 & G(2)
\end{array}\right)
$$

where $G(k): \mathbb{R}^{24} \rightarrow \mathbb{R}^{24}$ describes how the type of an infected individual of sex $k$ changes; 0 denotes the $24 \times 24$ zero matrix.

It follows from the assumptions that there are only six types with which an infected individual can be "born" (from the point of view of the infection): $(1, j ; 1)$ for males and $(1, j ; 2)$ for females, $1 \leqslant j \leqslant 3$. To calculate $R_{0}$ for each of the possible types at birth, we follow the remainder of the life of the corresponding individuals and count how many individuals of the different birth types he/she produces on the average. It was argued in [3] that $R_{0}$ is then given by the dominant eigenvalue of the matrix $M: \mathbb{R}^{6} \rightarrow \mathbb{R}^{6}$ (the so-called next-generation operator)

$$
\left(\begin{array}{cc}
0 & M_{2} \\
M_{1} & 0
\end{array}\right)
$$

where the $M_{k}=\left(m_{i j}(k)\right)_{1 \leqslant i, j \leqslant 3}, k \in\{1,2\}$, describe the average number of new cases caused by the different male (female) types among the different female (male) types, respectively. For these we can derive expressions

$$
\begin{aligned}
m_{i j}(1)= & -p_{i}(1) \beta\left(G(1)^{-1}\right)_{L(i, 0 ; 1) L(1, j ; 1)} \\
& -p_{i}(1) \rho(1)\left(G(1)^{-1}\right)_{L(i,-1 ; 1) L(1, j ; 1)} \\
m_{i j}(2)= & -p_{i}(2) \beta\left(G(2)^{-1}\right)_{L(i, 0 ; 2) L(1, j ; 2)} \\
& -p_{i}(2) \rho(2)\left(G(2)^{-1}\right)_{L(i,-1 ; 2) L(1, j ; 2)}
\end{aligned}
$$

for $1 \leqslant i, j \leqslant 3$ (see [3]). These formulas have the following interpretation. The second term of $m_{i j}(1)$ describes the expected number of first contacts (pair establishing), where the infection is successfully transmitted by an infected male who was "born" with type $(1, j ; 1)$, while he is of type $(i,-1 ; 1)$. The first term of $m_{i j}(1)$ describes the average number of females that become infected while a partner of an infected male with infection state $i$ who was "born" with type $(1, j ; 1)$. 
Next let us describe $G(k), k \in\{1,2\}$ :

$$
G(k)=\left(\begin{array}{cccc}
A_{1} & 0 & 0 & 0 \\
D_{1} & A_{2} & 0 & 0 \\
0 & D_{2} & A_{3} & 0 \\
0 & 0 & D_{3} & A_{4}
\end{array}\right)
$$

where 0 is the $6 \times 6$ zero matrix, $D_{j}=\operatorname{diag}\left(\theta_{j}\right)$, and $A_{i}, i \in\{1,2,3,4\}$, is given by

$$
\left(\begin{array}{cccccc}
a_{1}(i) & s_{i 0}\left(\mu_{0}+\sigma\right) & s_{i 1}\left(\mu_{1}+\sigma\right) & s_{i 2}\left(\mu_{2}+\sigma\right) & s_{i 3}\left(\mu_{3}+\sigma\right) & s_{i 4}\left(\mu_{4}+\sigma\right) \\
\left(1-p_{i}(k)\right) \rho(k) & a_{2}(i) & 0 & 0 & 0 & 0 \\
p_{i}(k) \rho(k) & p_{i}(k) \beta & a_{3}(i) & 0 & 0 & 0 \\
0 & 0 & \theta_{1} & a_{4}(i) & 0 & 0 \\
0 & 0 & 0 & \theta_{2} & a_{5}(i) & 0 \\
0 & 0 & 0 & 0 & \theta_{3} & a_{6}(i)
\end{array}\right) \text {, }
$$

where $a_{1}(i)=-\mu_{i}-\theta_{i}-\rho(k) ; a_{2}(i)=-\mu_{i}-\mu_{0}-\theta_{i}-\sigma-p_{i}(k) \beta$; $a_{3}(i)=-\mu_{i}-\mu_{1}-\theta_{i}-\theta_{1}-\sigma ; a_{4}(i)=-\mu_{i}-\mu_{2}-\theta_{i}-\theta_{2}-\sigma ; a_{5}(i)=$ $-\mu_{i}-\mu_{3}-\theta_{i}-\theta_{3}-\sigma ; a_{6}(i)=-\mu_{i}-\mu_{4}-\theta_{i}-\sigma$. For $A_{4}$ we have to read zero for $\beta$ and $\rho(k)$ because of the assumptions.

It is easily verified that $G(k)^{-1}$ can be expressed in the $6 \times 6$ matrices that constitute $G(k)$ as follows:

$$
\begin{aligned}
& G(k)^{-1} \\
& \quad=\left(\begin{array}{cccc}
A_{1}^{-1} & 0 & 0 & 0 \\
-D_{1} A_{2}^{-1} A_{1}^{-1} & A_{2}^{-1} & 0 & 0 \\
-D_{1} D_{2} A_{3}^{-1} A_{2}^{-1} A_{1}^{-1} & -D_{2} A_{3}^{-1} A_{2}^{-1} & A_{3}^{-1} & 0 \\
-D_{1} D_{2} D_{3} A_{4}^{-1} A_{3}^{-1} A_{2}^{-1} A_{1}^{-1} & D_{2} D_{3} A_{4}^{-1} A_{3}^{-1} A_{2}^{-1} & -D_{3} A_{4}^{-1} A_{3}^{-1} & A_{4}^{-1}
\end{array}\right) .
\end{aligned}
$$

The matrix $M$ can now be determined. For the special case $p_{2}(k)=0$, for $k \in\{1,2\}$, we find that

$$
M_{k}=\left(\begin{array}{ccc}
m_{11}(k) & 0 & m_{13}(k) \\
0 & 0 & 0 \\
m_{31}(k) & 0 & m_{33}(k)
\end{array}\right) .
$$

In terms of the elements of $G(k)^{-1}$ we can write

$$
m_{11}(k)=-p_{1}(k) \beta\left(G(k)^{-1}\right)_{2,3}-p_{1}(k) \rho(k)\left(G(k)^{-1}\right)_{1,3},
$$




$$
\begin{aligned}
& m_{13}(k)=-p_{1}(k) \beta\left(G(k)^{-1}\right)_{2,5}-p_{1}(k) \rho(k)\left(G(k)^{-1}\right)_{1,5}, \\
& m_{31}(k)=-p_{3}(k) \beta\left(G(k)^{-1}\right)_{14,3}-p_{3}(k) \rho(k)\left(G(k)^{-1}\right)_{13,3}, \\
& m_{33}(k)=-p_{3}(k) \beta\left(G(k)^{-1}\right)_{14,5}-p_{3}(k) \rho(k)\left(G(k)^{-1}\right)_{13,5},
\end{aligned}
$$

Then the spectral radius $r(M)=\sqrt{r\left(M_{1} M_{2}\right)}=\sqrt{r\left(M_{1}^{\prime} M_{2}^{\prime}\right)}$, where

$$
M_{k}^{\prime}=\left(\begin{array}{ll}
m_{11}(k) & m_{13}(k) \\
m_{31}(k) & m_{33}(k)
\end{array}\right), \quad k \in\{1,2\}
$$

see [3].

Finally we find that

$$
R_{0}=\frac{(a+d)+\sqrt{(a-d)^{2}+4 c b}}{2}
$$

with

$$
\begin{aligned}
& a:=m_{11}(1) m_{11}(2)+m_{13}(1) m_{31}(2), \\
& b:=m_{11}(1) m_{13}(2)+m_{13}(1) m_{33}(2), \\
& c:=m_{31}(1) m_{11}(2)+m_{33}(1) m_{31}(2), \\
& d:=m_{31}(1) m_{13}(2)+m_{33}(1) m_{33}(2) .
\end{aligned}
$$

\section{DESCRIPTION OF THE CALCULATIONS}

We define $N$ to be the average number of new sexual partners and $C$ the average number of sexual contacts that a newly infected individual will have in the remainder of his or her lifetime.

Let $\hat{r}$ be the right eigenvector of matrix $M$ corresponding to the dominant eigenvalue $R_{0}: M \hat{r}=R_{0} \hat{r}$. Then $\hat{r}$ can be interpreted as the asymptotic distribution of infected individuals over the six types at birth. We normalize $\hat{r}=\left(r_{1}, \ldots, r_{6}\right)^{T}$ to obtain the probability distribution of infected individuals over the six possible birth types $(1, j ; k), j \in\{1,2,3\}$, $k \in\{1,2\}$ (which we number $1-6$ ).

Denote by $N_{l}$ the average number of new sexual partners that a just-infected individual born with birth type $l \in\{1, \ldots, 6\}$ has during the 
remainder of his or her lifetime. Then

$$
N=\sum_{l=1}^{6} N_{l} r_{l}
$$

The numbers $N_{l}$ are given by

$$
N_{(1, j ; k)}=-\sum_{i=1}^{3} \rho(k)\left(G(k)^{-1}\right)_{L(i,-1 ; k) L(1, j ; k)}
$$

where $L$ describes the lexicographic ordering of types as explained in Section 2.

To calculate $C$, let $C_{l}$ denote the average total number of sexual contacts the individual will have after becoming infected as birth type $l \in\{1, \ldots, 6\}$. Then $C_{l}$ is given by

$$
C_{l}=N_{l}+\beta T_{l},
$$

being the sum of the number of "first contacts" and the average future time $T_{l}$ a newly infected individual with birth type $l$ will spend with a partner multiplied by the frequency $\beta$ of sexual contacts within a partnership. $T_{l}$ can be written as

$$
\begin{aligned}
T_{(1, j ; k)}=-\sum_{i=1}^{3}\{ & \left(G(k)^{-1}\right)_{L(i, 0 ; k) L(1, j ; k)}+\left(G(k)^{-1}\right)_{L(i, 1 ; k) L(1, j ; k)} \\
& \left.+\left(G(k)^{-1}\right)_{L(i, 2 ; k) L(1, j ; k)}+\left(G(k)^{-1}\right)_{L(i, 3 ; k) L(1, j ; k)}\right\} .
\end{aligned}
$$

The quantity $C$ is then given by the sum of the six $C_{l}$ 's weighted according to birth type:

$$
C=\sum_{l=1}^{6} C_{l} r_{l}
$$

We want to investigate $R_{0}$ as a function of the number $N$ of new sexual partners after infection. Of particular interest is the threshold value $N$ for which $R_{0}=1$. If we want to ensure uniqueness of this threshold value, to make the results comparable, we are not free to choose any combination of the pair formation parameters $\rho$ and $\sigma$. If $\sigma$ is allowed to increase while all other parameters are held constant, then 
the graph $R_{0}=f(N)$ need not be monotonically increasing, and therefore the threshold value may not be unique [4]. The reason for this lack of monotonicity is that for increasing separation rate, other parameters remaining constant, the infected individual spends less and less time in partnerships. Hence, the average total number of contacts will decrease, which in turn results in a smaller value of $R_{0}$. Therefore, to ensure monotonicity, and hence uniqueness of the threshold value, we keep the total number of contacts after infection $C$ constant when we vary $N$.

It is worthwhile to explain how the graphs of $R_{0}$ as a function of $N$ were made. In the numerical calculations we consider only the case that the sex ratio in the susceptible population is 1 . From the consistency requirement mentioned earlier, we then obtain that $\rho(1)=\rho(2)=: \rho$. We want to plot $R_{0}=f(N)$, for $N \in[1,20]$ say, with $C=\bar{c}$ a prechosen constant. Both $N$ and $C$ are functions of $\sigma$ and $\rho: N=g_{1}(\sigma, \rho)$ and $C=g_{2}(\sigma, \rho)$, say. If $\rho=\bar{\rho}$ has been chosen, then we can determine $\sigma=\bar{\sigma}$ such that $g_{2}(\bar{\sigma}, \bar{\rho})=\bar{c}$ [formally write $\bar{\sigma}=h(\bar{c}, \bar{p})$ ] by the secant method. Now calculate $R_{0}=f\left(g_{1}(h(\bar{c}, \bar{\rho}), \bar{\rho})\right)$, plot the result against $g_{1}(\bar{\sigma}, \bar{\rho})$, and choose a new $\bar{\rho}$.

\section{NUMERICAL RESULTS}

The following parameter values are held fixed throughout all computations: $\theta_{1}=4.0$ per year, $\theta_{2}=\theta_{3}=0.2$ per year; $\mu_{i}=0.02$ per year $(i \neq 4), \mu_{4}=0.5$ per year; $\beta=100$ per year; $C=500$. We assume that $s_{i j}=1$ for $i \in\{1,2,3\}, j \in\{0,1,2,3\}$ because in our model we disregard testing for seropositivity, and therefore infected individuals who do not have AIDS and whose current partners also do not have AIDS have no way of knowing that they are infected. Things are different if the current partner of our index case (the infected individual we follow) has AIDS. Then, if this partnership breaks up, our index case will know that he or she has a high probability of being infected. We choose $s_{i 4}:=0$, $i=\{1,2,3\}$. The remaining $s_{4 j}$ 's, $j \in\{0,1,2,3\}$, do not require special treatment. In these cases the index case himself has AIDS, and because of our assumptions this individual will then not have any sexual contacts or new sexual partners for the remaining time of his infectious period. The behavior of these individuals, and the value of $s_{4 j}$, will not influence $R_{0}$, nor does it influence the calculation of $N$ for the population. In our calculations below we have set $s_{4 j}=1$. One could argue that the value of $s_{i j}$ can depend on the reason (separation or death of the partner) that a partnership of the index case breaks up. However, as all these influences are unknown, there is really no point to such generality. 
In a series of four graphs, we evaluate the effect of the dependence of the infection probabilities per sexual contact on infection state and sex. We want to study four situations (the numbers correspond to the graphs in Figure 1):

(1) Infection state-dependent and sex-dependent $p_{i}$.

(2) Infection state-dependent and sex-independent $p_{i}$.

(3) Infection state-independent and sex-dependent $p_{i}$.

(4) Infection state-independent and sex-independent $p_{i}$.

We have to be careful in gauging the four situations if we want to be able to compare the results. We calibrate situations 2-4 using our choice for the most general situation 1 .

For situation 1 we choose $p_{1}(1)=0.05, p_{2}(1)=0.001, p_{3}(1)=0.01$, $p_{4}(1)=0$ for the male or female probabilities and $p_{1}(2)=0.025, p_{2}(2)=$ $0.0005, p_{3}(2)=0.005, p_{4}(2)=0$ for the female to male probabilities.

For situations 3 and 4 we first calculate duration of infectivity $D$ :

$$
D=\frac{1}{\mu_{1}+\theta_{1}}+\frac{\theta_{1}}{\left(\mu_{1}+\theta_{1}\right)\left(\mu_{2}+\theta_{2}\right)}+\frac{\theta_{1} \theta_{2}}{\left(\mu_{1}+\theta_{1}\right)\left(\mu_{2}+\theta_{2}\right)\left(\mu_{3}+\theta_{3}\right)} .
$$

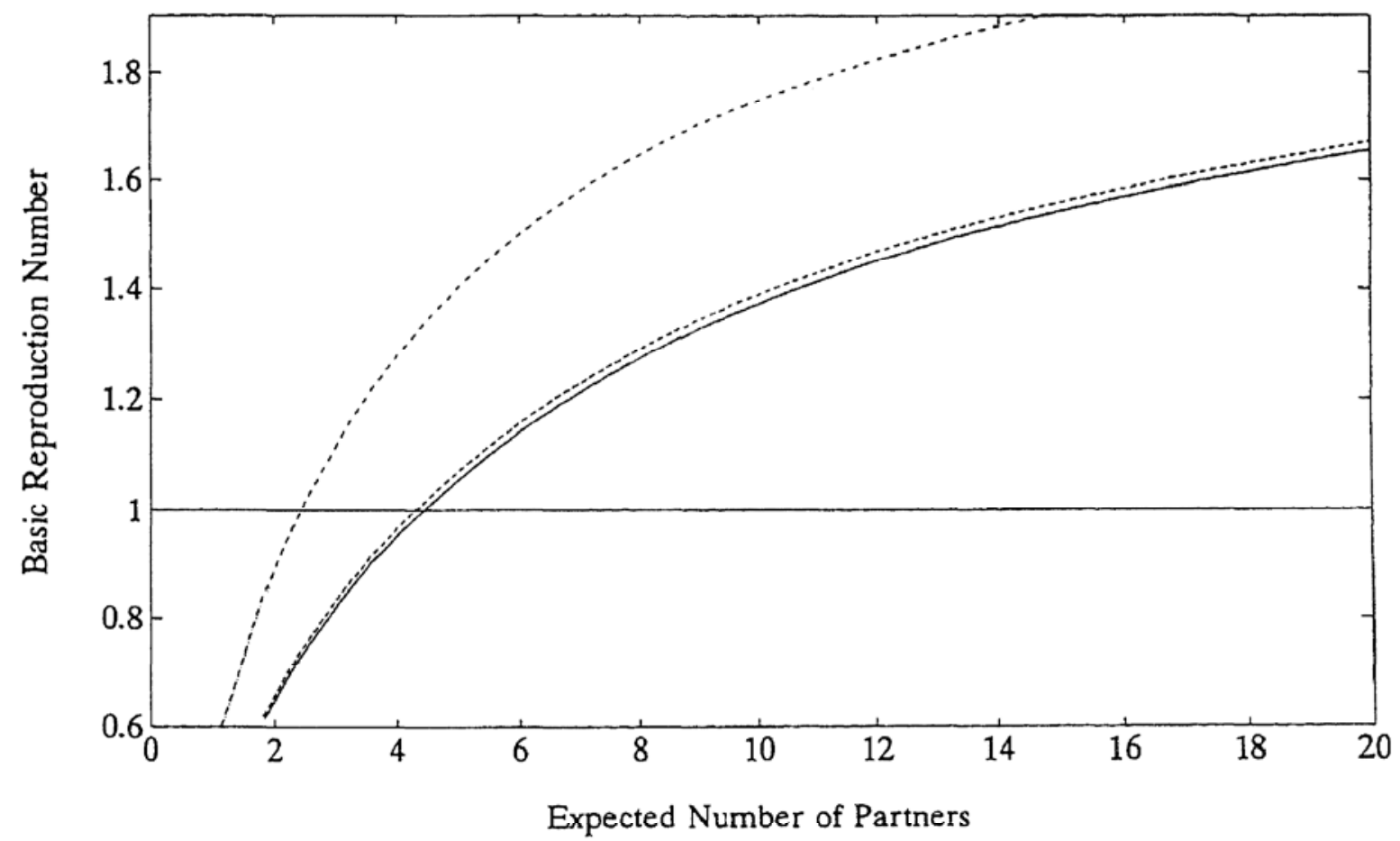

Fig. 1. The basic reproduction ratio $R_{0}$ as a function of the number $N$ of new partners during the infectious period for four different assumptions about the infection probabilities per contact. (-. - time- and sex-independent $(4) ;(\cdots)$ time-independent, sex-dependent (3); ( $-(-)$ time-dependent, sex-dependent (2); (-) time- and sex-dependent (1). For parameter values see text. 
Then the mean infectivity $\bar{p}$ is

$$
\begin{aligned}
\bar{p}=\frac{1}{D}( & \frac{p_{1}}{\mu_{1}+\theta_{1}}+\frac{p_{2} \theta_{1}}{\left(\mu_{1}+\theta_{1}\right)\left(\mu_{2}+\theta_{2}\right)} \\
& \left.+\frac{p_{3} \theta_{1} \theta_{2}}{\left(\mu_{1}+\theta_{1}\right)\left(\mu_{2}+\theta_{2}\right)\left(\mu_{3}+\theta_{3}\right)}\right) .
\end{aligned}
$$

We can use (1), with appropriate placing of $k$ 's, to calculate, from the original $p_{i}(k)$ 's, a $\bar{p}(1)$ for males and a $\bar{p}(2)$ for females. This leads to $\bar{p}(1)=0.00654$ and $\bar{p}(2)=0.00327$. We use these in situation 3. For situation 4 we take the geometric mean $\sqrt{\bar{p}(1) \bar{p}(2)}=0.00462$.

Finally, in situation 2, we take for $i=1,2$ the geometric mean over the sexes, $p_{i}=\sqrt{p_{i}(1) p_{i}(2)}$. This leads to $p_{1}=0.0354, p_{2}=0.0007$. Now, to gauge all situations we demand that $p_{1}, p_{2}$, and $p_{3}$ are such that $\bar{p}$ from (1) is equal to the geometric mean of $\bar{p}(1)$ and $\bar{p}(2)$. This requirement leads to $p_{3}=0.0071$.

For given values of $\rho$, a corresponding value for $\sigma$ was calculated to obtain a total of 500 contacts during the infectious period. For $\rho$ values ranging from 0.75 to 5.25 per year, the corresponding $\sigma$ values range from 0.36 to 4.35 per year in situations 1 and 2 and from 0.18 to 4.32 in situations 3 and 4 . The resulting four graphs are shown in Figure 1. The ordering of the four situations is

$$
R_{0}(1) \leqslant R_{0}(2) \leqslant R_{0}(3) \leqslant R_{0}(4) .
$$

We see from Figure 1 that the sex dependence does not appear to have a marked influence on $R_{0}$. This would suggest that the often-made distinction between "male to female" and "female to male" infection probabilities is not so relevant for questions involving the basic reproduction ratio. Dependence on the infection state, however, does have a marked influence on $R_{0}$, and the use of an infection state-independent infection probability would lead to an overestimation of the basic reproduction ratio. The effect of dependence on infection state on $R_{0}$ is larger for higher values of $N$. In any case, the numerical computations suggest that the introduction of variance in infectivity results in a decrease in the predicted $R_{0}$ value for a given value of $N$ if the average infectivity is kept constant. Repeating the calculations with different distributions of infectivity over the infection states while keeping the (weighted) integral over the entire infectious period constant did not lead to qualitatively different results. 
The threshold values $N^{*}$ for the number of partners during the infectious period of $D=8.88$ years satisfy the inequalities

$$
N^{*}(4)<N^{*}(3)<N^{*}(2)<N^{*}(1),
$$

where $N^{*}(4)=2.42, N^{*}(3)=2.49, N^{*}(2)=4.28$, and $N^{*}(1)=4.42$ [note that $N^{*}(1)$ is $83 \%$ larger than $N^{*}(4)$ ]. The critical number of partners $N^{*}$ can be even larger if one increases the variability of the infection probabilities. For $p_{1}=0.165$ and $p_{2}=p_{3}=0$, such that $\bar{p}$ remains the same, one gets $N^{*}=75.8$.

If we let $N$ tend to the maximum value that corresponds to the condition $C=500$ - in other words, if we set $N=500$ while letting $\sigma \rightarrow \infty$-we obtain the same value for all four curves: $R_{0}=2.31$. The curves differ primarily for intermediate values of $N$. This is easy to explain. For large values of $N$ they agree because the number of contacts per partner tends to 1 , so the infection probability per partner equals the infection probability per contact and the average infection probabilities all agree. Here we note that with respect to sex differences one has to take the geometric average, and with respect to time differences the integral over the infectious period. For small values of $N$, the curves agree because the number of contacts per partner is large so the infection probability per partner tends to 1 in all four cases.

If only individuals in the initial phase of the incubation period are infectious, then one has the intuitive feeling that $R_{0}$ will strongly depend on the length of this initial phase for a given average infectivity and fixed rates $\rho$ and $\sigma$ describing the formation and separation of pairs. If the infectious phase is shorter than the duration of a partnership plus the subsequent time between partnerships, then serial monogamy cannot sustain HIV transmission. This is illustrated in Figure 2, which shows $R_{0}$ as a function of the duration of the infectious phase, keeping the average infectivity fixed at 0.005 . The length of the incubation period is 10 years.

For $\sigma=\rho=1$ per year, we find that the index case acquires about 4.3 new partners with 93 contacts per new partner. For a constant infection probability we expect $R_{0}$ to be 1.60 according to the formula

$$
N\left(1-(1-\bar{p})^{c}\right)
$$

where $c$ denotes the number of contacts per partner. The corresponding values for $\sigma=\rho=2$ are 48 contacts per new partner, 9.3 partners, and $R_{0}=2.0$. Figure 2 shows $R_{0}$ as a function of the duration of the infectious phase. We see that $R_{0}>1$ would require a duration of about 5 years for $\sigma=\rho=1$ and 2.5 years for $\sigma=\rho=2$. This result may 


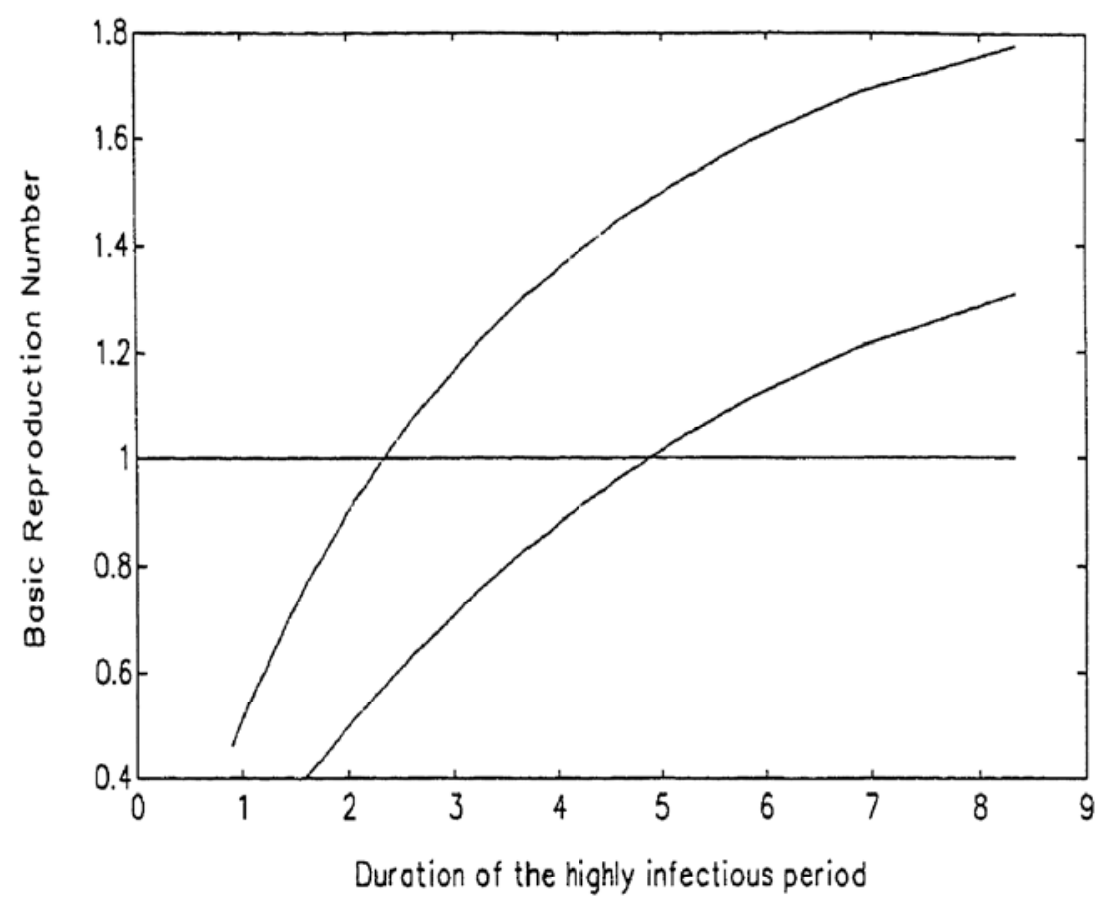

FIG. 2. The basic reproduction ratio $R_{0}$ as a function of the duration of the infectious phase in years, keeping the average infectivity per contact $\bar{p}$ fixed at 0.005 . The upper curve refers to an average duration of a partnership of half a year followed by an interval between partnerships of half a year. The corresponding durations for the lower curve are both 1 year.

provide one explanation for the slow spread of HIV in the heterosexual population in Europe outside the group of IV drug users.

The following argument demonstrates the plausibility that variability of infectivity during the infectious period decreases the infection probability per partner. Let $\omega(p)$ be the probability density function for the infection probability per contact $p$. In our model this distribution is concentrated on a finite number of different values. Let the probability-generating function for the number of contacts per partner be denoted by $F$. Then the infection probability per partner is given by

$$
1-\int_{0}^{1} F(1-p) \omega(p) d p
$$

since for a fixed number $n$ of contacts per partner the infection probability per partner is $1-(1-p)^{n}$ for a given infection probability $p$ per partner. If we expand $F$, we get

$$
\bar{p} c-(1 / 2)\left(\bar{p}^{2}+\sigma_{p}^{2}\right)\left(c^{2}-c+\sigma_{c}^{2}\right)+\cdots,
$$

where $\bar{p}$ is the average infectivity, $\sigma_{p}^{2}$ is the variance of the infectivity, 
$c$ is the mean number of contacts per partner, and $\sigma_{c}^{2}$ is the variance of the number of contacts per partner. In the epidemiological models that do not incorporate the possibility of longer lasting partnerships, exactly one contact takes place per partner. Therefore in those models $c=1$ and $\sigma_{p}^{2}=0$ and the infection probability per partner equals the average infection probability $\bar{p}$ per contact. The expansion given above indicates that the decrease in the infection probability depends on both the variability of infectivity and the variability of the number of contacts per partner. A similar argument was used by Dietz and Tudor [5] in describing the effects of concurrent partnerships on the calculation of $R_{0}$.

K. D. was supported in part by NATO grant (D.890350), and D. W. T. by the Deutsche Forschungsgemeinschaft (Di 308/5-1); J. A. P. H. was supported by his wife.

\section{REFERENCES}

1 S. P. Blythe and R. M. Anderson, Variable infectiousness in HIV transmission models, IMA J. Math. Appl. Med. Biol. 5:181-200 (1988).

2 O. Diekmann, J. A. P. Heesterbeek, and J. A. J. Metz, On the definition and the calculation of the basic reproduction ratio $R_{0}$ in models for infectious diseases in heterogeneous populations, J. Math. Biol. 28:365-382 (1990).

3 O. Diekmann, K. Dietz, and J. A. P. Heesterbeek, The basic reproduction ratio for sexually transmitted diseases, Part 1. Theoretical considerations, Math. Biosci. 107:325-339 (1991).

$4 \mathrm{~K}$. Dietz, The role of pair formation in the transmission dynamics of HIV, in Sesquicentennial Invited Paper Sessions, M. H. Gail and N. L. Johnson, Eds., American Statistical Association, Alexandria, Va., 1989, pp. 609-621.

$5 \mathrm{~K}$. Dietz and D. W. Tudor, Triangles in heterosexual HIV transmission, in AIDS Epidemiology: Methodological Issues, N. P. Jewell, K. Dietz, and V. T. Farewell, Eds., Birkhäuser, Boston, 1992, pp. 143-155.

6 J. A. Jacquez, C. P. Simon, J. Koopman, L. Sattenspiel, and T. Perry, Modeling and analyzing HIV transmission: the effect of contact patterns, Math. Biosci. 92:119-199 (1988). 